\title{
Cardiovascular Dynamics - Multiple Time Scales, Oscillations and Noise
}

\author{
Aneta Stefanovska $^{* \dagger}$, Andriy Bandrivskyy ${ }^{\dagger}$ and Peter V.E. McClintock ${ }^{\dagger}$ \\ ${ }^{*}$ Group of Nonlinear Dynamics and Synergetics, Faculty of Electrical Engineering, \\ University of Ljubljana, Ljubljana, Slovenia \\ ${ }^{\dagger}$ Department of Physics, Lancaster University, Lancaster, UK
}

\begin{abstract}
Modelling the cardiovascular system (CVS) presents a challenging and important problem. The CVS is a complex dynamical system that is vital to the function of the human organism, and it reflects numerous different states of health and disease. Its complexity lies in a combination of oscillatory modes spanning a wide frequency scale that can synchronize for short episodes of time, coupled with a strong stochastic contribution. Motivated by these properties, we discuss the problem of characterising dynamics when there is a combination of oscillatory components in the presence of strong noise and, in particular, where the characteristic frequencies and corresponding amplitudes vary in time. We show that, where there are several noisy oscillatory modes, the slower modes are difficult to characterise because the length of the recorded time series is inevitably limited in real measurements. We argue that, in the case of strong noise combined with a limited observation time, such oscillatory dynamics with several modes may appear to manifest as a $1 / f$-like behaviour. We also show that methods of time-frequency analysis can provide a basis for characterising noisy oscillations, but that a straightforward characterisation of multi-scale oscillatory dynamics in the presence of strong noise still remains an unsolved problem.
\end{abstract}

\section{INTRODUCTION}

Nature abounds with rhythms of diverse origin. The day and the night rhythmically exchange, influencing the rhythmicity of light- and temperature-dependent physiological processes. But natural clocks seem to be adaptive. As the length and intensity of the daylight change within a year, the basic frequencies of many natural rhythms are modulated and thus vary in time.

One of the most vital rhythmical functions for humans, and for higher animals generally, is the beating of the heart. The heart is a part of the cardiovascular system (CVS) which serves to keep the blood in continuous motion. The CVS rhythmically distributes nutrients and oxygen to every cell and takes away the products of their metabolism. It consists of the heart, lung, and a network of contractile vessels that are actively involved in the regulation of blood pressure and blood flow. The flow of blood is regulated with respect to the needs of each cell of the body, as well as to the spontaneous fluctuations arising from naturally occurring perturbations to the CVS. Thus the heart frequency is not constant, but varies within certain limits, adapting to the current state and needs of the organism.

The CVS is an extremely complex system, and it reflects the different states of health or disease. How best to parameterize CVS dynamics using appropriate cardiovascular signals, measured noninvasively, has been a continuing challenge over the last twenty 
years. In spite of intensive research, an optimal characterization able unambiguously to describe the dynamics of the CVS, and reliably to distinguish between its states, has yet to be found. In particular, we would like to establish a way of detecting changes due to diseases in their early stages, before symptoms become obvious. The characterization involves two major questions -

1. How to define the appropriate level of complexity at which the system can best be characterized and modelled?

2. What is the underlying dynamics that best characterize its properties?

The first problem involves understanding the structure and function of the CVS, i.e. the underlying physiological background. The second problem is usually approached through the analysis of cardiovascular time series. The recent development of noninvasive sensors based on electrical, optical, and auditory detection of cardiovascular functions, of powerful computers, and of methods of nonlinear and stochastic time series analysis, has motivated huge interest in understanding the dynamics of the cardiovascular system. However, the complex fluctuations which we can observe and measure in the blood flow, blood pressure and heart rate, are yet to be understood.

It has been established that signals, derived from the human CVS contain several oscillatory components $[1,2,3,4,5,6]$, independent of the measurement site and function. On a time scale of around one minute, the dynamics of the blood distribution system can be characterized by five oscillatory components with slowly varying frequencies and amplitudes $[7,8,9,10]$. The frequency and amplitude variations are reminiscent of phenomena observed in coupled oscillators. Moreover, another phenomenon characteristic of coupled oscillators was observed - the synchronization of two of the rhythms: the cardiac and respiratory $[11,12,13,14,15,16]$. One possibility, therefore, is to characterize cardiovascular signals in terms of a model consisting of five coupled autonomous noisy oscillators $[17,18]$.

The other approach comes from analyses of the statistical properties of heart rate variability (HRV) [19, 20, 21, 22, 23], and considers the chaotic behaviour [24, 25], scaling properties [26, 27, 28] and multifractal characteristics [23] detected in the HRV signal. HRV was considered to result from $1 / f$ dynamics and it was suggested that the state of the system defines the scaling properties of the power spectrum of the HRV and that the state can be classified in terms of the slope in the log-log representation of its power spectrum.

Where, then, is the origin of the difference in these two approaches to CVS dynamics? Is the dynamics primarily oscillatory - spanning multiple time scales, or is it mainly stochastic, or is it a combination? Studies concerned with the stochastic nature of the system are mainly based on analyses of the HRV signal. Those concerned with its oscillatory nature are based on analyses of blood flow and blood pressure, as well as of the HRV signal. They are motivated by the physiological importance of the local regulatory mechanisms for blood flow and blood pressure, which manifest as low frequency oscillations $(<0.05 \mathrm{~Hz})$ apparently reflecting the vascular and endothelial immune and cytotoxic reactions.

In this paper we illustrate problems that arise in the analysis of noisy time-varying oscillations from a finite data set, and we show that they may lead to differences in interpretation. We first analyze two cardiovascular signals, HRV and blood flow, and then apply 
exactly the same analysis to a model signal whose dynamics is completely known. For illustration several methods of time series analysis are applied: discrete Fourier transform (DFT), time averaged DFT, wavelet transform using the Morlet wavelet, time averaged wavelet transform, and detrended fluctuation analysis (DFA).

\section{TIME SERIES AND THEIR CHARACTERIZATION}

\section{Cardiovascular signals}

In this section we present analyses of cardiovascular time series. A set of cardiovascular signals, i.e. the electrical activity of the heart (ECG), respiratory movements of the lung, blood pressure, and peripheral blood flow, was simultaneously recorded from a healthy, relaxed subject for 30 minutes using the procedure described in [9]. In our discussion we will consider, first, the HRV "signal" derived from the ECG as an estimate of the instantaneous cardiac frequency defined between two successive R-peaks and, secondly, the skin blood flow signal. The signals were sampled at $400 \mathrm{~Hz}$ for precise detection of R-peaks. After generation of the HRV, they were resampled to $10 \mathrm{~Hz}$ for analysis. The resultant signals are presented in figures 1(a) and 2(a) respectively.
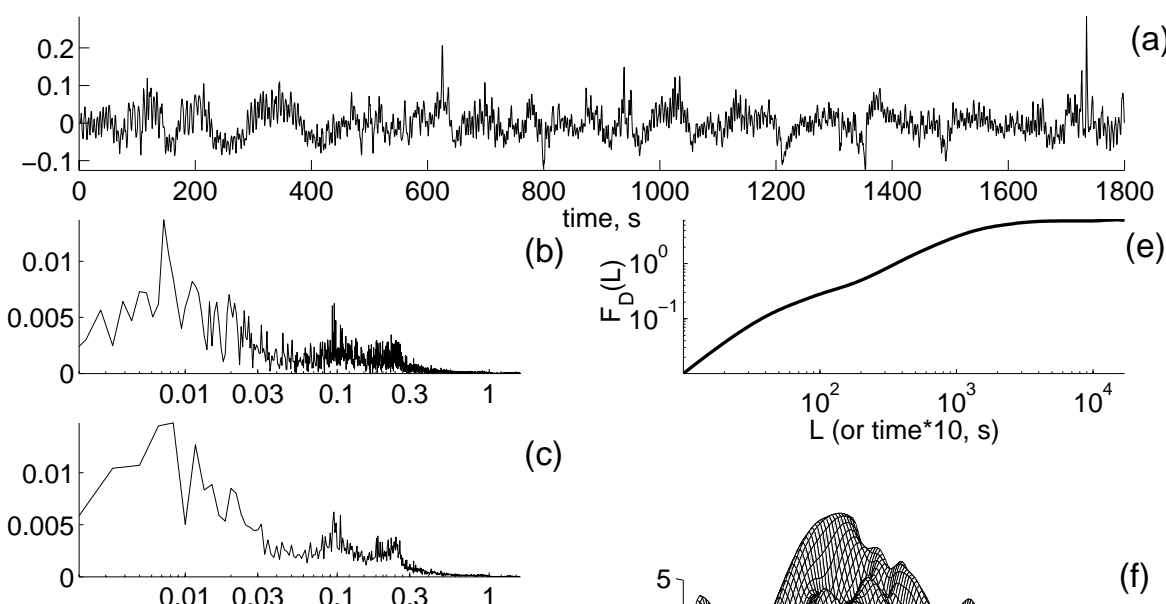

(c)

(a)
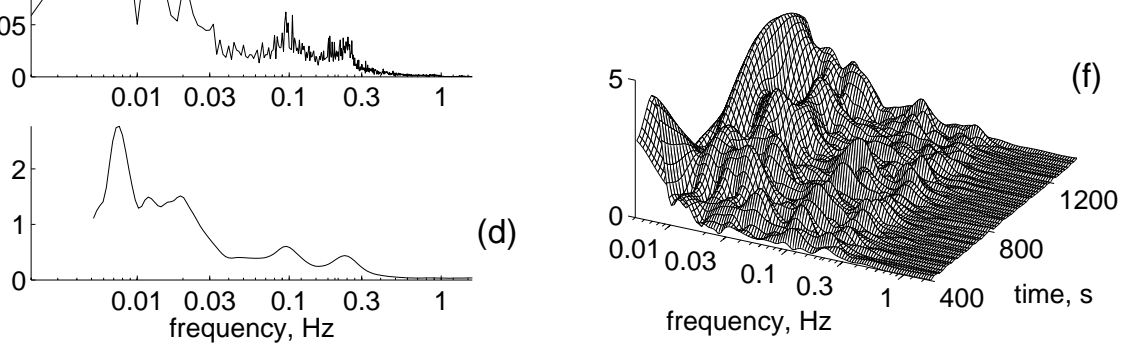

FIGURE 1. (a) The HRV signal and its (b) DFT, (c) time-averaged DFT, (d) time-averaged wavelet transform, (e) detrended fluctuation function and (f) its wavelet transform. The variations in (a) are in seconds around the mean value $(1 \mathrm{~s})$ that was subtracted for the analyses shown below. 

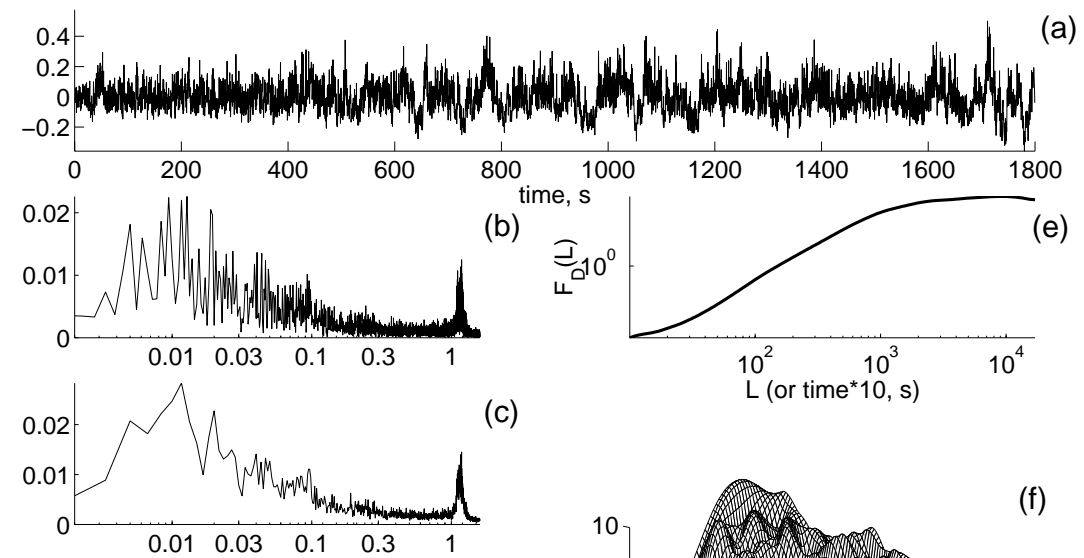

(c)

a)
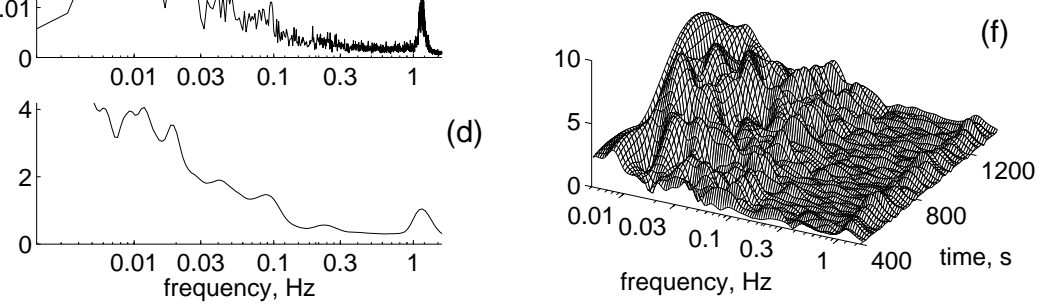

FIGURE 2. (a) The skin blood flow signal and its (b) DFT, (c) time-averaged DFT, (d) time-averaged wavelet transform, (e) detrended fluctuation function and (f) time-frequency projection of the wavelet transform.

Spectral analysis reveals the presence of low frequency components in both signals; note that, by definition, the HRV signal cannot contain the $(\sim 1 \mathrm{~Hz})$ cardiac frequency itself. However, markedly different interpretations of the very low frequency components are possible. One may think of oscillatory components which undergo strong complex modulation by noise and from the rest of the system, or one can study the scaling properties of the power spectra which can be classified by the $\alpha$ coefficient given by detrended fluctuation analysis (DFA) [21, 22]. As demonstrated in figures 1(e) and 2(e) both cardiovascular signals manifest $1 / f$-like behavior.

\section{Model time series}

Let us now consider a Van-der-Pol oscillator using the same methods of analysis. Figure 3(a) presents a time series obtained from one oscillator of the system

$$
\ddot{x}_{i}+\varepsilon_{i}\left(1-x_{i}^{2}\right) \dot{x}_{i}+w_{i}^{2} x_{i}=D_{i} \xi(t),
$$

where $x_{i}$ is the coordinate of the $i$-th oscillator and $\xi(t)$ is white Gaussian noise of intensity $D_{i}$. The length of the time series, the integration time step and the characteristic frequencies are all chosen to mach the statistical properties of the cardiovascular time 
series and the conditions under which they are analysed. Results are shown both in the absence of noise (left) and with strong noise (right). In the absence of noise the system


(b)
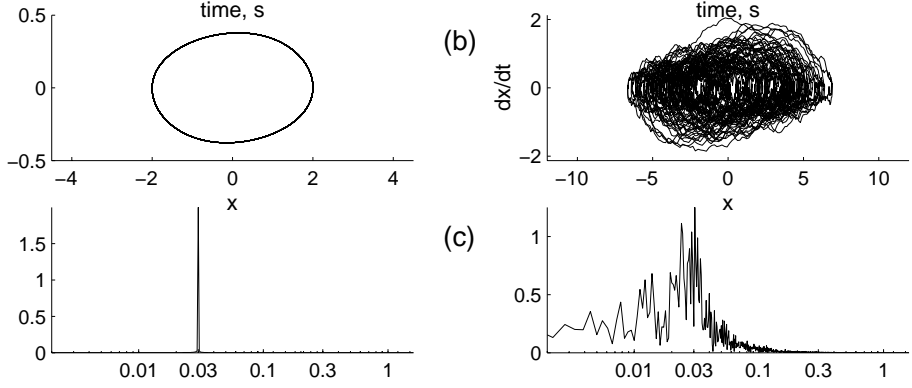

(c)
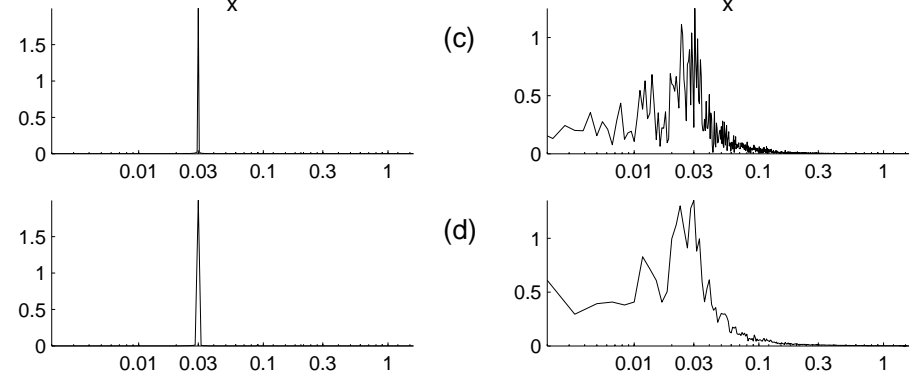

(d)
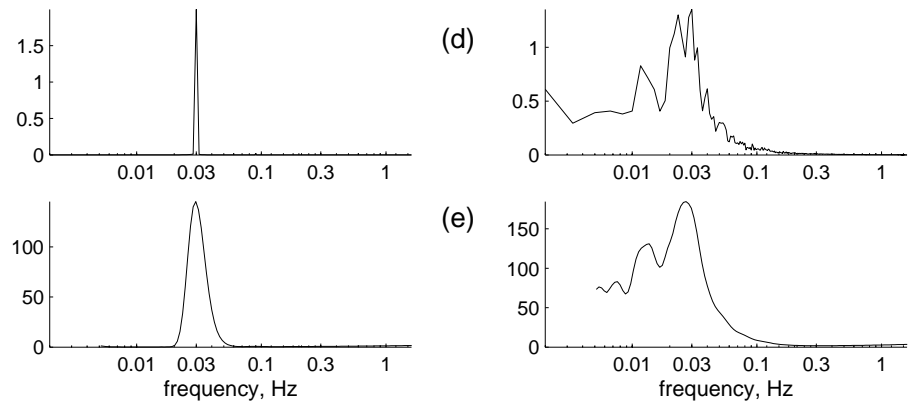

(e)
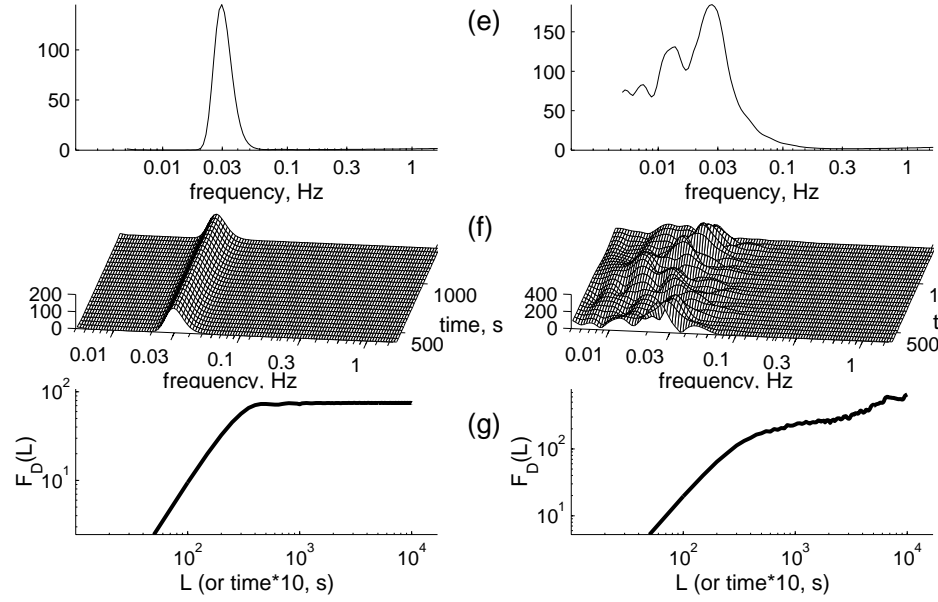

(f)

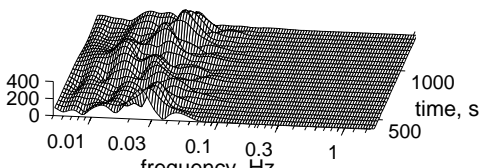

(g)

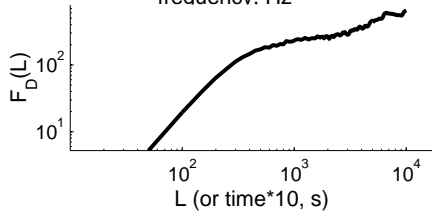

FIGURE 3. (a) A signal of one noise-free Van-der-Pol oscillator with frequency of $0.03 \mathrm{~Hz}$ (left), and in presence of strong noise (right). (b) Phase portrait, (c) DFT, (d) time-averaged DFT, (e) time averaged wavelet transform and (f) its wavelet transform and (g) detrended fluctuation function for noise-free (left) and noisy oscillator (right). $L$ in $(\mathrm{g})$ is the window length.

(1) possesses a stable periodic orbit (limit cycle) and produces an almost harmonic signal for small $\varepsilon_{i}$. Strong noise applied to the oscillator results in significant variations of its instantaneous amplitude and frequency, strikingly similar to the behaviour already observed in cardiovascular signals. In the absence of noise the problem of characterising 
the dynamics of a single oscillator from time series is of course trivial. Each of the existing methods, either in phase space, or in the time, frequency, or time-frequency domains, reconstructs its properties unambiguously. The addition of strong noise, however, transforms this trivial problem into one that remains unsolved. Note that the phase portrait of the single noisy oscillator can no longer be characterised by a circle and no obvious origin can be determined. The DFA then results in a curve that may be interpreted as $1 / f$-like, although the scaling interval(s) cannot easily be defined. A more precise characterisation may be attempted by spectral methods, and by time-frequency analysis in particular. Here, however, we face difficulties in frequency determination. The higher the frequency is, the better we are able to define it, as there will be a larger number of cycles within the observation period. For the slower oscillations, however, with fewer complete cycles, the effect of noise is inevitably more pronounced. Note that the choice of the window length represents a compromise between time and frequency localisation. The actual window for the wavelet transform in our case was chosen to include 5 periods of the corresponding frequency being estimated.

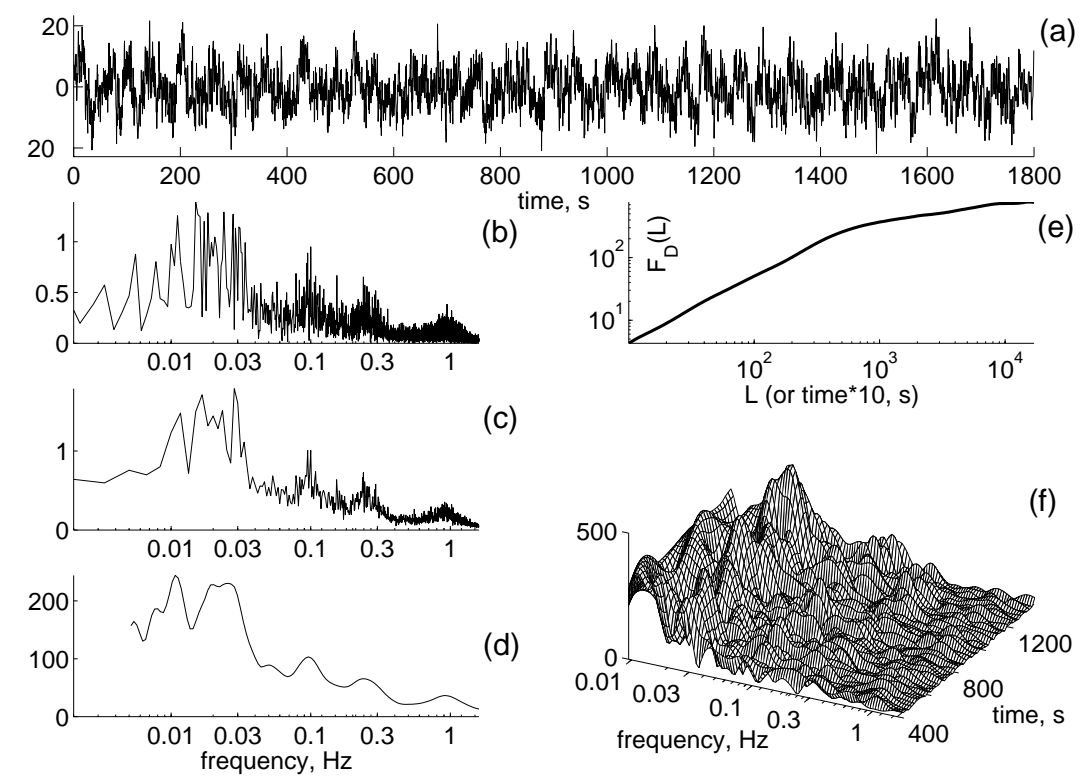

FIGURE 4. (a) The model signal and its (b) DFT, (c) time averaged DFT, (d) time averaged wavelet transform, (e) detrended fluctuation function and (f) its wavelet transform.

Now let us consider a more complex model with five noisy oscillators with characteristic frequencies $f_{i}=\{1,0.26,0.1,0.03,0.018\} \mathrm{Hz}$. The choices of the number of oscillators, and their frequencies, were motivated by the idea that CVS can be modelled as a system of five coupled oscillators, each corresponding to a different CVS activity (cardio, respiratory, neurogenic, myogenic and metabolic) [9]. We scale the parameters $w_{i}, \varepsilon_{i}$ and $D_{i}$ in order to get exactly the same oscillators but acting on different time scales. So if $k$ is a scaling factor so that $w_{i}=k w_{1}$, then $\varepsilon_{i}=k \varepsilon_{1} D_{i}=k^{2} D_{1}$. The time 
series in figure 4(a) are obtained as $x=\sum_{i} x_{i}$ for parameters $\varepsilon_{1}=0.4$ and $D_{1}=1000$. Note that all five oscillators (1) are identical, so that they produce identical time series in a statistical sense, but scaled in time and in frequency. Note also that in reality the cardiovascular oscillators are coupled. In our model we use noise to introduce the effect of couplings. In this way, although the model is complex, e.g. consists of five modes, we are still able to treat it both analytically and numerically.

Spectral analyses using frequency and time-frequency methods are presented in figures 4(b)-4(e). Note that the wavelet transform has better statistical averaging (averaging in time and frequency domain). However, as already noted, optimal resolution in time and in frequency cannot be obtained simultaneously. The Morlet wavelet allows a compromise with good frequency resolution in the low frequency (LF) domain and a good time resolution in the high frequency (HF) domain. It allows the best estimation of the lowest frequency peaks compared to the results obtained by DFT and time averaged DFT. Yet, as can be seen from figure 4, the two spectral peaks lowest in frequency are not well resolved in the spectra. That means that the available time of observation, which is chosen to include about 30 realizations of the slowest oscillation, is insufficient for appropriate estimation of the LF oscillatory components due to poor statistical averaging. Figure 4(e) gives the DFA function of the model signal in 4(a). Again it shows $1 / f$-like behavior with two different scaling regions. We note that DFA cannot provide information about the underlying oscillatory dynamics.

\section{CONCLUSION}

We have discussed the problem of characterising oscillatory dynamics from time series in the presence of strong noise, introduced in such a way as to produce time-variability of the characteristic frequencies as observed for the cardiovascular oscillations. Using model time series that mimic the main properties of the cardiovascular signals we show that, when the noise is strong and the frequency variability is large, the underlying dynamics cannot be determined unambiguously. Nonetheless, time-frequency methods have the potential advantage over DFA and phase-space techniques that they can in principle separate the different oscillatory components, and can thus provide information specific to particular physiological processes.

The time-variable characteristic oscillations can be taken as indicating of a nonautonomous system. A time-frequency, or time-phase representation is therefore needed for characterisation of the corresponding dynamics. The wavelet transform, as a timefrequency method, enables the instantaneous frequencies to be traced as they evolve in time. Where several oscillatory components are present, however, and because the time of observation in reality is inevitably finite, we face difficulties in estimation of the lower oscillation frequencies. The longer their period in comparison to the total observation time, the more obtrusive the noise becomes. The lower frequencies may thus be misestimated as the result of poor statistical averaging. We note that the problem of identifying characteristic frequencies in the presence of noise is widespread, because biological systems are characterised by inherently noisy oscillatory dynamics. 


\section{ACKNOWLEDGMENTS}

This work has been supported by the Slovenian Ministry of Education, Science and Sport, Joy Welch Trust (UK), the EPSRC (UK) and INTAS.

\section{REFERENCES}

1. Hyndman, B. W., Kitney, R. I., and Sayers, B. M., Nature, 233, 339-341 (1971).

2. Peňáz, J., Automedica, 2, 135-141 (1978).

3. Akselrod, S., Gordon, D., Ubel, F. A., Shannon, D. C., C., B. A., and Cohen, R. J., Science, 213, 220-222 (1981).

4. de Boer, R. W., Karemaker, J. M., and Strackee, J., Psychophysiology, 22, 147-155 (1985).

5. Camm, A. J., Malik, M., Bigger, J. T., and et al., Circulation, 93, 1043-1065 (1996).

6. Di Renzo, M., Parati, G., Pedotti, A., and Zanchetti, A. E., Frontiers of Blood Pressure and Heart Rate Analysis, IOS Press, Amsterdam, 1997.

7. Stefanovska, A., and Krošelj, P., Open Syst. and Inf. Dyn., 4, 457-478 (1997).

8. Kvernmo, H. D., Stefanovska, A., Bračič, M., Kirkeøen, K.-A., and Kvernebo, K., Microvasc. Res., 56, 173-182 (1998).

9. Stefanovska, A., and Bračič, M., Contemporary Physics, 40, 31-55 (1999).

10. Bračič, M., McClintock, P. V. E., and Stefanovska, A., "Characteristic frequencies of the human blood distribution system," in Stochastic and Chaotic Dynamics in the Lakes, edited by D. S. Broomhead, E. A. Luchinskaya, P. V. E. McClintock, and T. Mullin, American Institute of Physics, Melville, New York, 2000, pp. 146-153.

11. Kenner, T., Passenhofer, H., and Schwaberger, G., Pflügers Archiv., 363, 263-265 (1976).

12. Hildebrandt, G., "The autonomous time structure and its reactive modifications in the human organism," in Temporal Disorder in Human Oscillatory System, edited by L. Rensing, U. an der Heiden, and M. C. Mackey, Springer, Berlin, 1987, pp. 160-174.

13. Raschke, F., "Coordination in the circulatory and respiratory systems," in Temporal Disorder in Human Oscillatory System, edited by L. Rensing, U. an der Heiden, and M. C. Mackey, Springer, Berlin, 1987, pp. 152-158.

14. Schäfer, C., Rosenblum, M. G., and Kurths, J., Phys. Rev. E, 60, 857-870 (1999).

15. Bračič Lotrič, M., and Stefanovska, A., Physica A, 283, 451-461 (2000).

16. Stefanovska, A., Haken, H., McClintock, P. V. E., Hožič, M., Bajrović, F., and Ribarič, S., Phys. Rev. Lett., 85, 4831-4834 (2000).

17. Stefanovska, A., Bračič, M., Strle, S., and Haken, H., Physiol. Meas., 22, 535-550 (2001).

18. Stefanovska, A., Luchinsky, D. G., and McClintock, P. V. E., Physiol. Meas., 22, 551-564 (2001).

19. Kobayashi, M., and Musha, T., IEEE Trans. Biomed. Eng., 29, 456-457 (1982).

20. Peng, C. K., Mietus, J., Hausdorff, J. M., Havlin, S., Stanley, H. E., and Goldberger, A. R., Phys. Rev. Lett., 70, 1343 (1993).

21. Hausdorff, J. M., and Peng, C. K., Phys. Rev. E, 54, 2154 (1996).

22. Stanley, H. E., Amaral, L. A. N., Goldberger, A. L., Havlin, S., Ivanov, P. C., and Peng, C. K., Physica $D, 270,309-324$ (1999).

23. Ivanov, P. C., Amaral, L. A. N., Goldberger, A. L., Havlin, S., Rosenblum, M. G., Struzik, Z. R., and Stanley, H. E., Nature, 399, 461-465 (1999).

24. Babloyantz, A., and Destexhe, A., Biol. Cybern., 58, 203-211 (1988).

25. Poon, C. S., and Merrill, C. K., Nature, 389, 492-495 (1997).

26. Amaral, L. A. N., Goldberger, A. L., Ivanov, P. C., and Stanley, H. E., Phys. Rev. Lett., 81, 2388-2391 (1998).

27. Ashkenazy, Y., Ivanov, P. C., Havlin, C. K., Peng, A. L., Goldberger, A. L., and Stanley, H. E., Phys. Rev. Lett., 86, 1900-1903 (2001).

28. Bernaola-Galván, P., Ivanov, P. C., Amaral, L. A. N., and Stanley, H. E., Phys. Rev. Lett., 87, 168105 (2001). 
Copyright $\odot 2003$ EBSCO Publishing 
Copyright $\odot 2003$ EBSCO Publishing 
Copyright of AIP Conference Proceedings is the property of American Institute of Physics and its content may not be copied or emailed to multiple sites or posted to a listserv without the copyright holder's express written permission. However, users may print, download, or email articles for individual use. 